\title{
Beam Hopping Loss of Communication Satellite between Ground Relay Station Propagation
}

\author{
Deng Yaosheng \\ Northwestern Polytechnical University \\ YaoshengDeng@yeah.net
}

Keywords: Radio Propagation; Communication Satellite

\begin{abstract}
Communication satellites play an important role in daily life and war. Covering the ground and realizing the propagation between relay stations is one of the important performances of communication satellites. It is of great engineering and military significance to construct a universal and efficient coverage feature simulation method and to give accurate coverage performance evaluation indicators. This paper mainly studies the coverage of communication satellites on the ground: various coverage geometric models are established and simulated; various constraints of communication propagation process are discussed; and it is important to evaluate the coverage of communication satellites and the propagation of relay stations. Performance indicators, and these indicators were statistically analyzed by SPSS.
\end{abstract}

\section{Determination of the Best Working Frequency}

To establish reliable shortwave communication, it's essential to choose the appropriate working frequency seriously. After considering the propagation condition which mostly affects the sky- wave propagation, the operating frequency of HF communications can be predicted. For the reason that the "sky wave" propagation conditions vary from the number of sunspots, thus the number of sunspots can be used as a significant factor in the propagation of shortwave. And this significant factor can measure the "Limit frequency curve", which propagates between $E$ layer and $F_{2}$ layer under the conditions of the maximum and minimum number of sunspots. The "limit frequency curve" indicates the change curve of the frequency of reflection between $E$ layer and $F_{2}$ layer in 24 hours of a day, which can confirm the highest available frequency band of the normal propagation. 


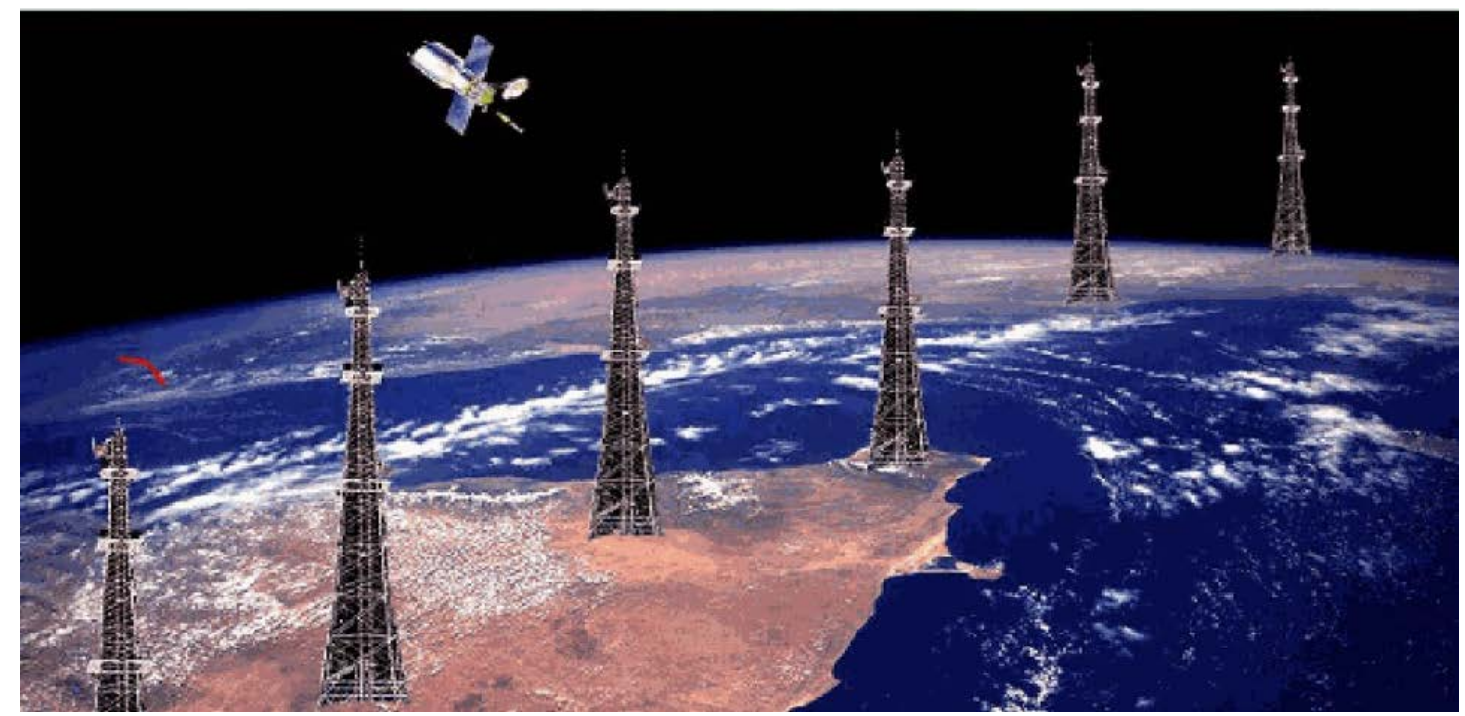

Figure 1 The coverage of communication satellites and the propagation of relay stations

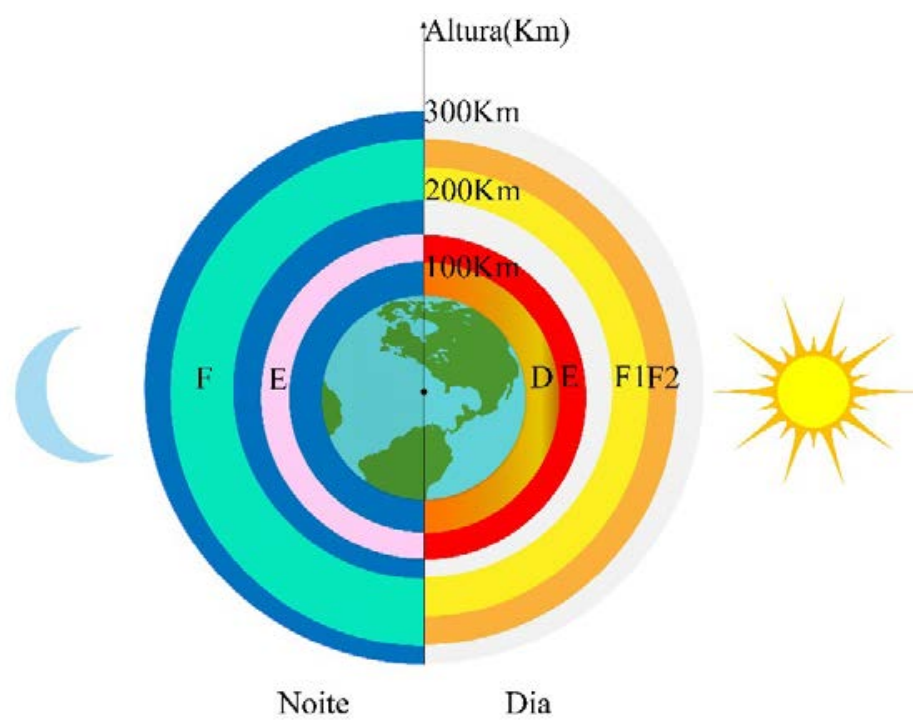

Figure 2 Ionosphere stratification

The ionosphere is structured in Fig.2, which is discretized by the electron density and the collision frequency. As the sun is the major source of ionization, the layers vary from day to night.

$>\quad$ Layer E Maximum Available Frequency Test

$$
\operatorname{EMUF}(t, n)=f_{0} E \times \sec \left(i_{110}\right) M H Z
$$

Where: $f_{0} E$ is the critical frequency. $i_{110}$ is the incidence angle of radio waves at $110 \mathrm{~km}$

\section{Layer $F_{2}$ Maximum Available Frequency Test}

$$
F_{2} \operatorname{MUF}(t, n)=\left[1+\left(\frac{C_{d}}{C_{3000}}\right)(B-1)\right] \times f_{0} F_{2}+\frac{f_{H}}{2}\left(1-\frac{d_{h}}{d_{\max }}\right) M H Z
$$

Where: $d_{\max }$ is the maximum hop length for layer $F_{2} . C_{d}=0.071, B=1.2$. 


\section{Signal Reflection Model for Geographical Environment}

Combined with the reflection model for a calm ocean, since free space loss and ionospheric loss are not affected by the route of transmission, the greatest difference between land and ocean transmission loss is $L_{g}$.

Next we will study the propagation loss on the ground and the reception intensity of the terrestrial transmission.

To accurately characterize the paths of ionospheric waves, it is necessary to determine the relationship between the following geometric parameters.

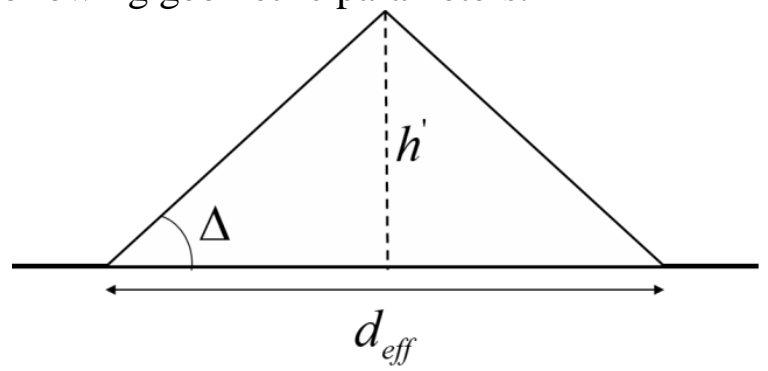

Figure 3 Geometry of the trajectory the wave that reflects the ionosphere

According to Fig 3, the angle of incidence $\psi$ is given by:

$$
\Delta=\tan ^{-1} \frac{2 h^{\prime}}{d_{\text {eff }}}
$$

\subsection{Specular Reflection}

The interference of the direct radiation with the reflected radiation causes the electric field to fluctuate, and it can strongly change the signal at the receiving antenna compared with the received signal in free space.

The total electric field, which is the sum of the direct ray and the reflected ray, can be described as follows:

$$
E_{d}[1+|\Gamma| \exp (j \Delta \phi)]
$$
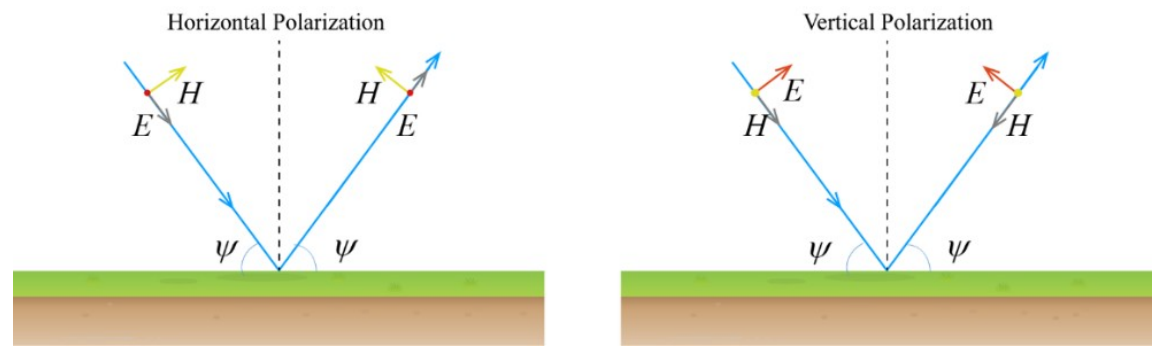

Figure 4 Reflection in horizontal and vertical polarizations

Where: $E_{d}$ is the free space field. $\Gamma$ is the ground reflection coefficient. $\Delta \phi$ is the phase difference.

The reflection coefficients for horizontal and vertical polarizations are given respectively by: 


$$
\begin{aligned}
& P H: \Gamma_{h}=\frac{\sin \Delta-\sqrt{n^{2}-\cos ^{2} \Delta}}{\sin \Delta+\sqrt{n^{2}-\cos ^{2} \Delta}} \\
& P V: \Gamma_{v}=\frac{n^{2} \sin \Delta-\sqrt{n^{2}-\cos ^{2} \Delta}}{n^{2} \sin \Delta+\sqrt{n^{2}-\cos ^{2} \Delta}}
\end{aligned}
$$

The difference between the direct and reflected paths of the signal wave determines the phase difference between the signals

$$
\begin{gathered}
\Delta \phi=\arg [\Gamma]-2 \pi \frac{\Delta r}{\lambda} \\
\Delta r=r_{d}-r_{r}
\end{gathered}
$$

Where: $r_{r}$ is the distance from the signal to the reflection point. $r_{d}$ is the distance from the signal sent point to the signal receive point.

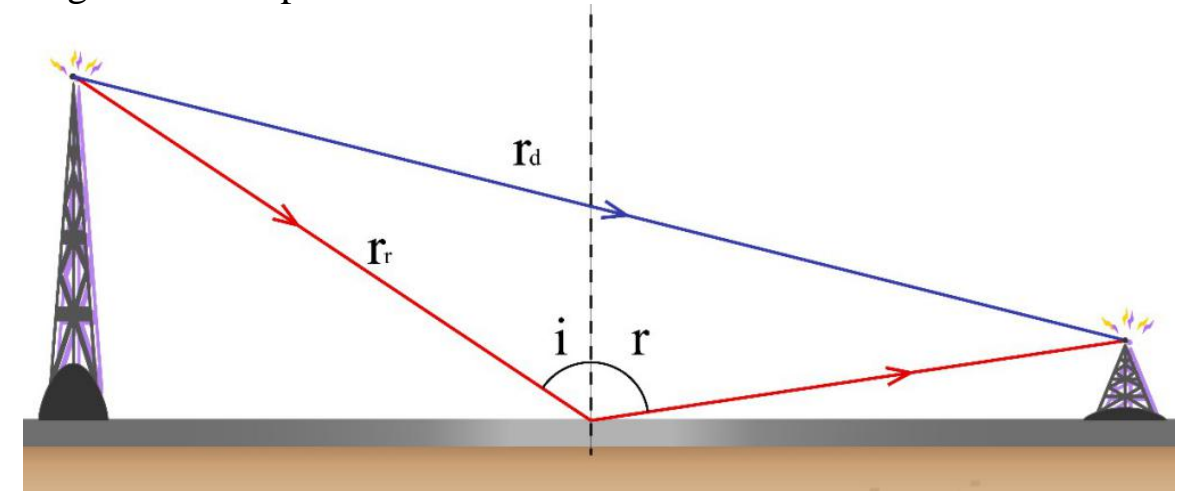

Figure 5 Direct and reflected rays

\subsection{Diffuse Reflection}

$$
\begin{gathered}
E=E_{d}\left[1+|\Gamma| e^{-g^{2}} \exp (j \Delta \phi)\right] \\
g=\frac{4 \pi}{\lambda} h_{e} \sin \psi
\end{gathered}
$$

Where: $g$ is the phase difference relative to any two points on the ground, $h_{e}$ is the roughness of the ground.

\subsection{Rough Ground Attenuation}

The attenuation of the signal reflected in the rough is given by:

$$
L_{g g}=|\Gamma|^{2} e^{-g^{2}} D(D=1, \text { due to the assumption of flat earth })
$$

Where: $L_{g g}$ is the land transmission loss. As a result, the corrected basic transmission loss $L_{b}$ is as follow:

$$
L_{b}=L_{b f}+L_{a}+L_{g g}+L_{p}(d B)
$$


Combining with the Okumura-Hata model, we finally come to the empirical formula of signal wave propagation on land.

$$
L_{b}=69.55+26.16 \lg f-13.82 \lg h_{t}-a\left(h_{r}\right)+\left(44.9-6.55 \lg h_{t}\right)(\lg d)^{\gamma}+K
$$

Where: $a\left(h_{r}\right)$ is the receive antenna height correction factor. $h_{t}, h_{r}$ are the effective height of the transceiver antenna. $\gamma$ is the distance correction factor.

$K$ is described as follows:

$$
K= \begin{cases}-\left(2 \lg (f / 28)^{2}\right)+54 & \text { suburbs } \\ -\left(4.78 l^{2} g f-18.33 \lg f+40.94\right) & \text { openland } \\ -\left(4.78 l^{2} g f-18.33 \lg f+40.94\right)+5.5 & \text { quasi openland } \\ -\left(\lg (f / 28)^{2}\right)-2.39(\lg f)^{2}+9.17 \lg f-23.17 & \text { countryside } \\ -(-5.7+0.024 \Delta h+6.96 \lg \Delta h)-\left(9.5 \lg \mathrm{h}_{1}-7.2\right) & \text { hills, } \Delta h \geq 15, h_{1} \geq 1 \\ -(-5.7+0.024 \Delta h+6.96 \lg \Delta h)+7.2 & \text { hills, } \Delta h \geq 15, h_{1}<1 \\ 0 & \text { hills, } \Delta h<15\end{cases}
$$

\section{References}

[1] JYRKI T. J. PENTTINEN, The Telecommunications Handbook Engineering Guidelines for Fixed, Mobile and Satellite Systems.[A]Electrical \& Electronics Engineering-Communication Technology. 2015(01)

[2] Hu Honglin,Zhu Jinkang. A BHA Communication Scheme and Its Performance Analysis [J]. Chinese High Technology Letters,2003(08):8-13 